DEPARTMENT OF THE INTERIOR

UNITED STATES GEOLOGICAL SURVEY

\title{
SLOPE MAP, CONNECTICUT VALLEY URBAN AREA, CENTRAL NEW ENGLAND
}

\section{By United States Geological Survey}

